\title{
The concept of illness behaviour: culture, situation and personal predisposition ${ }^{1}$
}

The concept of illness or disease refers to limited scientific models for characterizing constellations of symptoms and the conditions underlying them. The concept of illness behaviour, in contrast, describes the ways in which people respond to bodily indications and the conditions under which they come to view them as abnormal. Illness behaviour thus involves the manner in which individuals monitor their bodies, define and interpret their symptoms, take remedial action, and utilize sources of help as well as the more formal health care system. It also is concerned with how people monitor and respond to symptoms and symptom change over the course of an illness and how this affects behaviour, remedial actions taken, and response to treatment. The different perceptions, evaluations and responses to illness have, at times, a dramatic impact on the extent to which symptoms interfere with usual life routines, chronicity, attainment of appropriate care and cooperation of the patient in treatment. Variables affecting illness behaviour usually come into play well before any medical scrutiny and treatment (Mechanic, 1978).

A crucial premise in the study of illness behaviour is that illness, as well as illness experience, is shaped by sociocultural and social-psychological factors, irrespective of their genetic, physiological or other biological bases. A way from the research laboratory, illness is often used to achieve a variety of social and personal objectives having little to do with biological systems or the pathogenesis of disease. The boundaries of illness and its definitions are potentially very broad, and the illness process can be used to negotiate a range of cultural, social and personal tensions in the home, at work, and in the community at large.

Cultural definitions, social development and personal needs shape the experience of illness and meanings attributed to physical factors that serve as its basis. While the magnitude, severity, persistence and character of symptoms affect and establish limits for personal and social definitions, there is considerable variability in what is perceived, how it is defined, the interventions that are considered and used, requests for support and special consideration, and illness outcomes.

A major gap in our knowledge concerns those processes that lead persons exposed to similar stressors to respond differentially at a physical, psychological or social level. While the neuropsychological bases of alternative patterns of expressing distress remain uncertain, many research workers have ingeniously demonstrated that there may be conversions among different levels of experience and that substitutability among levels of response is to some unknown degree operative (Graham, 1972).

While the superiority of longitudinal data are generally recognized for establishing clear causal links, the role of such data in the study of illness behaviour is particularly crucial. Much confusion and conflicting findings in the field arise from cross-sectional multivariate analyses that ignore the essential dynamic features of the illness experience and coping associated with it.

Take, for example, the issue of denial in illness. Much effort is wasted in attempting to assess whether denial in the case of myocardial infarction, cancer, etc. is a useful defensive reaction. Viewing illness behaviour as a dynamic process, however, reveals that the question is poorly conceptualized and that efforts to answer it are often misguided. Illness experience is part of a continuing process of adaptation in which the individual's efforts to cope are linked in various ways with the particular

\footnotetext{
1 This paper was first presented at the First International Conference on Clinical and Social Aspects of Illness Behaviour, The Royal Adelaide Hospital, Australia, August 1984. Address for correspondence: Dr David Mechanic, Health and Health Care Services Research Coordinating Council, Room 203, Murray Hall, New Brunswick, NJ 08903, USA.
} 
dimensions of the threat, and both change over time. Thus, at the initial stage of threat following the occurrence of a serious heart attack, where life may be in the balance, denial may effectively serve to reduce distress and to increase the probabilities of survival. Denial at the time of early symptoms, in contrast, may be life-threatening. As patients emerge from the critical period and must face the longer term challenges of their illness and rehabilitation efforts, denial may further restrict access to essential information and needed care. A more refined and realistic approach is to examine how denial functions differently at varying stages of illness, and its consequences as it interacts with other factors.

Much attention is now being devoted to patients' appraisals of their symptoms, the assumptions they make about causes, and how responses to medical advice are conditioned by the 'naïve' theories that patients use to understand their bodily responses. Studies consistently demonstrate major inconsistencies between physicians' expectations and assumptions and patients' responses, resulting in poor communication and difficulties in treatment. Leventhal and colleagues $(1980,1986)$ find, for example, that while physicians assume that patients with hypertension cannot make judgements of their blood pressure, many patients feel confident in their abilities to assess when their blood pressure is high or low and they adjust their treatment regimens accordingly. Knowing the physician's view, they withhold their assumptions or information about modifications of regimen. Understanding illness theories used by patients thus offers potential for improved communication, more appropriate therapeutic instruction, better treatment regimens, and enhanced adherence to medical advice.

One of the most persistent observations in the epidemiological literature concerns the substantial relationships among reports of physical morbidity, psychological symptoms and self-assessments of health. In one sense, these relationships may be a result of dualism in our language and conceptualizations; there is only a single body but we talk in parallel languages about it (Graham, 1967). At least three central hypotheses have emerged about the relationship between physical and psychological symptoms, each correct to some degree. First, the enormity of serious physical illness causes psychological distress and perhaps also some psychiatric illnesses among vulnerable persons. Secondly, it has been known for decades that major and persistent psychological stress predisposes individuals to physical illness (Dohrenwend \& Dohrenwend, 1974, 1981). While there has been active controversy as to whether these effects are specific to a limited set of diagnoses (Graham, 1972) or are relevant to all diagnoses and bodily systems, few seriously doubt that the 'psychosomatic hypothesis' is in some sense valid. The problem consists less in the validity of the insight and more in our incapacities to conceptualize such relationships in a manner promoting increased understanding and improved interventions. A third hypothesis is that certain predispositions of the individual, whether shaped by biology, culture or particular psychological histories, increase the sensitivity or vulnerability of individuals to both physical and psychological symptoms. In our current work we focus on one such predisposition - the tendency to introspect, for example, to be particularly concerned with one's thoughts and feelings. This predisposition is shaped by both culture and social relations, and interacts with varying aspects of social situations.

\section{DETERMINANTS OF ALTERNATIVE EXPRESSION PATTERNS}

People experience many troubles and tensions that culminate in a variety of adaptations, including physical illness, psychological disorder, and a wide range of attack, escape and risk-taking behaviour. Biology and culture are clearly major limiting factors on how such internal states are expressed. However, a large proportion of what is viewed as illness in modern society appears to be the end point of a process with a variety of alternative pathways. It is estimated that as many as $50 \%$ of patients entering medical care have symptoms and complaints that do not fit the International Classification of Diseases, and many others are motivated in seeking care by problems and symptoms other than those with which they present (White, 1970). This suggests the limitations of medical knowledge to some extent, but it also reflects the fact that going to a doctor is part of a process 
of illness behaviour, iqvolving such factors as felt need, perception, appraisal, definition, attribution of cause, and decision-making.

To the epidemiologist, who begins with a medical definition of a case and not with the processes that lead to its social definition, there are many anomalous findings. Why, for example, are rates of depression, neurotic disturbance, demoralization, and the use of medication relatively high among women, and alcoholism, hard drug use, and violence particularly high among men? Are these independent observations, or is there an underlying process leading to alternative pathways of expression? Why, among populations such as the Chinese, are affective expressions of depression uncommon but the somatic components relatively frequent? Why are rates of suicide among Blacks in the United States relatively low, but rates of homicide so high? An understanding of such questions requires inquiries into culture, social situations and personal predispositions.

A wide range of findings indicates that, in many instances, individuals are unaware of the factors which influence their decisions and actions, even when questioned immediately after the event, and they often deny that such influences affected them even when such influences are identified (Nisbett \& Wilson, 1970). Observations of this kind are more comprehensible if we take note of the fact that effective adaptation is often facilitated by the normalization of disruptive or uncomfortable situations and maintenance of the perception of a regular flow of activities. Successful coping often requires the illusion that major changes are limited in impact and that the continuity of the individual persists without major alteration (Mechanic, 1970; Davis, 1963). In contrast to crisis, successful coping involves barely perceptible changes in which the response to challenge becomes part of the ordinary. To be too self-aware of change, and how one is being influenced, is itself stressful (Mechanic, 1962). While people may be altered dramatically over time in dealing with illness or difficult life events, it often serves their interests to perceive that little has changed and to believe that their behaviour arises from acceptable and readily understandable causes. By examining the factors which contribute to distortion, students of illness behaviour enhance their understanding of their subject matter, since distortion is part of the process of defining, struggling with, and coping with potential illness challenges.

\section{SOCIOCULTURAL INFLUENCES}

The extraordinary differences among cultures in characterizations of illness, conceptions of causation, and modes of treatment are substantially documented in the anthropological literature (Harwood, 1981). Even in modern populations one finds an interesting blend of sophisticated scientific ideas and folk wisdom learned from close peers and through intuitive processes. Such cultural content affects the recognition and conceptualization of symptoms, the vocabularies for communication, modes and content of expression, and the range of remedial efforts. Even limited historical study reveals how substantially expressions of distress and illness are transformed from one era to another.

The expression of illness through psychological and social vocabularies is a relatively modern phenomenon coexistent with a growth of personal self-awareness and broad self-expression. Female hysteria, for example, characterized by fainting, conversion reactions and 'kicking about' has virtually disappeared in the urban modern environment, although vestiges of the disorder are still seen occasionally in isolated rural cultures that are relatively psychologically unsophisticated and repressive. One interpretation of the disappearance of hysteria is that it no longer brings a sympathetic response from the social environment and is suspect not only among physicians but also among sophisticated persons more generally (Veith, 1970). But, while the dramatic histrionics of hysteria are rarely seen, more mundane expressions of the somatization of distress are ubiquitous and constitute a major load on the medical care system in all nations.

In any historical period the prevailing norms and ideologies encourage or constrain the selection among alternative modes of tension reduction. People facing personal troubles draw on existing sociocultural conceptions of the nature of these troubles and what one might do about them. At any point in time there may be more or less social consensus surrounding the definition of the problem, 
beliefs about cause, and conceptions of possible remedies. These shape how people view their troubles and what actions they contemplate. Personal tension, for example, may be defined alternatively as resulting from physical illness, personality deficiencies, moral dilemmas, cultural inhibitions, or social exploitation. The availability of reference groups that adhere to particular definitions and provide social support is essential to the maintenance of particular interpretations (Greenley et al. 1976; Kadushin, 1966, 1969). The development of women's consciousness groups in Western nations is a case in point. Prior to such developments, many women experiencing distress and dissatisfaction came to psychiatrists complaining of unhappiness and feelings of inadequacy as wives, mothers and women. The problem was viewed primarily as a unique personal problem resulting from the client's personality and social development. With the growth of the women's movement such feelings are increasingly seen as a consequence of constraining role expectations, exploitative role relationships and blocked opportunities to achieve aspirations, and it is not difficult to find many women reinforcing this conception and providing social support. Thus personal distress is increasingly being redefined as a commonly shared social problem. Similar redefinitions are increasingly applied by the aged, the handicapped and homosexuals. This is not to suggest that such groups do not have serious personal problems or handicaps. But such examples demonstrate that the construction of health and disease is a social process, often arbitrary in conceptualization (Dubos, 1959).

\section{THE SOCIAL SITUATION}

Illness behaviour arises in response to circumstances that challenge the ongoing homeostasis. People are extraordinarily adaptive, but some changes in the situation, whether arising within the organism or from external factors, induce self-consciousness and appraisal, and require assessments about the nature of the problem, its causes and the strategies to be initiated. These responses are both cognitive and affective, concerned with understanding the changes and maintaining emotional equilibrium.

Illness behaviour is more than a psychological response among persons faced with a situation calling for assessment. It also arises in response to troubling social situations and may serve as an effective means of achieving release from social expectations, as an excuse for failure, or as a way of obtaining a variety of privileges including monetary compensation. Moreover, the physician and other health personnel may be an important source of social support and may be particularly important for patients lacking strong social ties. A vague complaint of illness may be one way of seeking reassurance and support through a recognized and socially acceptable relationship when it is difficult for the patient to confront the underlying problem in an unambiguous way without displaying weaknesses and vulnerabilities contrary to expected and learned behaviour patterns. Balint (1957) and others have noted that the presenting symptoms may be of no special importance, but serve to establish the relationship between the patient and the doctor.

A vast number of doctor-patient contacts involve symptoms and illnesses that are widely distributed in the population and that are more frequently untreated than treated (White et al. 1961). Thus the decision to seek care is frequently a result of contingencies surrounding the perception of symptoms. Perceptions of oneself as ill and seeking care may provide self-justification when potential failure poses much greater symbolic threats to the individual's self-esteem than the process of being ill or dependent (Cole \& Lejeune, 1972).

A related issue is the difficulty experienced by some patients of differentiating symptoms of psychological origin from symptoms of particular diseases. Many illnesses, or effects of medications prescribed for dealing with them, produce feelings comparable with those associated with stress and psychopathology. Such symptoms as fatigue, restlessness and poor appetite, for example, may result either from depression or from an acute infectious disease. When both occur concurrently, patients may attribute the effects of one to another. There is an indication, for example, that long convalescence from acute infectious disease may result from the attribution of symptoms caused by depression to the acute condition (Imboden et al. 1961). This complicates not only the patient's recovery but also the physician's perception and management of the patient. 


\section{PERSONAL PREDISPOSITIONS}

The variability in patient behaviour in a given subculture, despite the similarity of symptoms, also reflects major differences in psychological orientations and predispositions. At a simple level, people vary in their tolerance for discomfort, in the knowledge, information and understanding which they have about the illness process, and in the specific ways in which bodily indications affect needs and ongoing social roles. People seem to vary a great deal in their subjective response to pain and discomfort, although there appears to be much less difference in physical thresholds. Much research has demonstrated that pain has an important subjective component, and there is no clear relationship between the amount of tissue damage and the degree of discomfort reported by the patient (Beecher, 1959; Melzack, 1973). What people know, believe, and think about illness, of course, affects what symptoms they think are important, what is viewed as more or less serious, and what one should do.

We are engaged at present in research on one specific predisposition - introspectiveness (a tendency to think about onself, and one's motivations and feelings) - which we believe is fundamental to understanding appraisal and illness behaviour (Mechanic, 1983). Over the years there have been numerous efforts to measure personal traits which characterize individuals who display an exaggerated illness dependency. During the height of dominance of psychodynamic ideas in psychiatry such individuals were commonly characterized as 'neurotic' and, more recently, as 'worried wells', but neither concept provides an adequate conceptualization of such personal traits or the processes by which exaggerated illness patterns develop.

The concept of enduring personal traits has increasingly been called into question. There was little evidence that personal characteristics were stable across situations or over time. While the difficulty of demonstrating stability may, in part, be methodological (Epstein, 1979), current data suggest that it is more appropriate to speak of predispositions or orientations which may be operative only under certain conditions.

Introspection appears to be an important orientation in the illness behaviour process. Results from research programmes investigating such varied issues as self-esteem, objective self-awareness, pain response, behaviour and health, private self-consciousness and ego defence processes all support the hypothesis that attention to self increases the prevalence of reported psychological and physical symptoms and negative self-evaluations (Mechanic, 1983).

I first made this observation more than 20 years ago, but did not fully appreciate its significance. We had asked mothers of young children to keep detailed illness diaries for themselves and other family members, requiring responses to a specific symptom inventory each day. We had some difficulty in gaining cooperation and, when we questioned mothers who were reluctant, some indicated that the attention to symptoms required by completing the diary, made them feel worse. What was so evident phenomenologically to the individuals involved has now been clearly established in a variety of laboratory studies under controlled conditions.

In a variety of studies examining introspection we find that individuals with such an orientation report more physical and psychological distress, are more upset by stressful life events, and use more medical, psychiatric and other helping services (Mechanic, 1979, 1980; Greenley \& Mechanic, 1976; Mechanic \& Hansell, 1983). This orientation, we believe, is conditioned by sociocultural factors and childhood socialization, and it may be triggered by particular situational events (Mechanic, 1972). More recently, we have found that individuals with an introspective orientation are more reactive to interpersonal adversities affecting significant others, while those less directed to their internal states show less distress (Mechanic \& Hansell, 1983).

The fact that people cope much of the time without awareness is a central point in understanding personal and social adaptation. To become aware, to become self-conscious, is an indication of more than a routine problem, a greater challenge, a break in the flow of normal activity. People can at any time be confronted with serious illness or personal tragedy. But it is not psychologically economical to worry about what one cannot predict or control, and individuals maintain a sense of invulnerability by inattention to potential threat (Janis, 1951; Mechanic, 1972). Too much inattention 
or denial distracts attention from essential information acquisition and planning, but we maintain our comfort by a considerable filtering of potentially threatening information.

Individuals who are more introspective probably know themselves better, and perhaps have a better understanding of the influences that affect them, but they also appear more uncomfortable with themselves and their life situations. They are more prone to react to threatening situations and more likely to define many common, self-limited bodily sensations as symptoms. While introspectiveness, properly guided, may be associated with valued consequences - such as creativity, sensitivity and empathy with others, and artistic expression - it also appears to exaggerate the experience of distress and illness.

Introspectiveness is only one of many personal inclinations or traits that may interact with perceptions of threat, coping and the illness experience. Such traits may profoundly affect perceptions and response, the course of the illness experience, and the quality of adaptation. They also may affect the propensity to view oneself as ill, the degree of personal suffering experienced, and the degree to which common bodily signals are conceptualized in a threatening way.

In sum, illness behaviour involves a complex interaction between the quality of bodily dysfunction, the sociocultural and psychological orientations brought by individuals to their situation, and the unique demands of the immediate social context. Epidemiological studies show that most people can elicit symptoms comparable with those most commonly presented in medical interactions. The challenge is to gain a better understanding of the question posed so clearly by Michael Balint in helping general practitioners to understand their patients' behaviour. Why has the patient chosen to emphasize this time, and this set of symptoms? The fact that similar, and even more serious, symptoms were evident on other occasions when no comparable behaviour took place remains the core of the puzzle we strive to understand.

DAVID MECHANIC

\section{REFERENCES}

Balint, M. (1957). The Doctor, His Patient and the Illness. International Universities Press: New York.

Beecher, H. K. (1959). Measurement of Subjective Responses: Quantitative Effects of Drugs. Oxford University Press: New York. Cole, S. \& Lejeune, R. (1972). Illness and the legitimation of failure. American Sociological Review 37, 347-356.

Davis, F. (1963). Passage Through Crisis. Polıo Victims and Their Families. Bobbs-Merrill Co.: Indianapolis.

Dohrenwend, B. S. \& Dohrenwend, B. P. (eds.) (1974). Stressful Life Events: Their Nature and Effects. Wiley Interscience: New York.

Dohrenwend, B. S. \& Dohrenwend, B. P. (eds.) (1981). Stressful Life Events and Their Contexts. Rutgers University Press: New Brunswick, NJ.

Dubos, R. (1959). Mirage of Health. Utopias, Progress, and Biological Change. Harper and Bros: New York.

Epstein, S. (1979). The stability of behavior. I: On predicting most of the people much of the time. Journal of Personality and Social Psychiatry 37, 1097-1126.

Graham, D. T. (1967). Health, disease, and the mind-body problem: linguistic parallelism. Psychosomatic Medicine 29, 52-71.

Graham, D. T. (1972). Psychosomatic medicine. In Handbook of Psychophysiology (ed. N. S. Greenfield and R. A. Sternbach), pp. 839-924. Holt, Rinehart and Winston. New York.

Greenley, J. R. \& Mechanıc, D. (1976). Social selection in seeking help for psychological problems. Journal of Health and Soctal Behaviour 17, 249-262.

Harwood, A. (ed.) (1981). Ethnicity and Medical Care. Harvard University Press: Cambridge, Mass.

Imboden, J. B., Canter, A. \& Cluff, L. (1961). Symptomatic recovery from medical disorders. Journal of the American Medical Association 178, 1182-1184.

Janis, I. L. (1951). Air War and Emotional Stress: Psychological Studies of Bombing and Civilian Defense. McGraw-Hill: New York.
Kadushin, C. (1966). The friends and supporters of psychotherapy: on social circles in urban life. American Sociological Review 31, 786-802.

Kadushin, C. (1969). Why People Go to Psychiatrists. Atherton: New York.

Leventhal, H., Meyer, D. \& Nerenz, D. (1980). The common-sense representation of illness danger. In Medical Psychology, Vol. 2 (ed. D. Rachman), pp. 7-30. Pergamon: New York.

Leventhal, H., Prohaska, T. H. \& Hirschman, R. S. (1986) Preventive health behavior across the life-span. In Preventing Health Risk Behaviors and Promoting Coping with Illness, Vol. 8 (ed. J. C. Rosen and L. J. Solomon). University of New England: Hanover, N. H. (in the press).

Mechanic, D. (1962). Students Under Stress: A Study in the Social Psychology of Adaptation. Free Press: New York. (Republished by the University of Wisconsin Press with a new introduction, 1978.)

Mechanic, D. (1970). Some problems in developing a social psychology of adaptation to stress. In Social and Psychological Factors in Stress (ed. J. E. McGrath), pp. 104-123. Holt, Rinehart and Winston: New York.

Mechanic, D. (1972). Social psychologic factors affecting the presentation of bodily complaints. New England Journal of Medicine 286, 1132-1139.

Mechanic, D. (1978). Medical Sociology (second edn). Free Press: New York.

Mechanic, D. (1979). Development of psychological distress among young adults. Archives of General Psychiatry 36, 1233-1239.

Mechanic, D. (1980). The experience and reporting of common physical complaints, Journal of Health and Social Behaviour 21, 146-155.

Mechanic, D. (1983). Adolescent health and illness behaviour: hypotheses for the study of distress in youth. Journal of Human Stress 9, 4-13 
Mechanic, D. \& Hansell, S. (1983). Adolescent introspectiveness, psychological distress, and physical symptoms. Presented at the 78th Annual Meeting of the American Sociological Association, Detroit, September.

Melzack, R. (1973). The Puzzle of Pain: Revolution in Theory and Treatment. Basic Books: New York.

Nisbett, R. N. \& Wilson, T. D. (1970). Telling more than we can know: verbal reports of mental processes. Psychological Review 84, 231-259.
Veith, I. (1970). Hysteria: The History of a Disease. University of Chicago Press: Chicago.

White, K. L. (1970). Evaluation of medical education and health care. In Community Medicine: Teaching, Research, and Health Care (ed. W. Lathem and A. Newbery), pp. 241-270. Appleton-CenturyCrofts: New York.

White, K. L., Williams, T. F. \& Greenberg, B. G. (1961). The ecology of medical care. New England Journal of Medicine 265, 885-892. 